\title{
[Invited] Elastic Optical Interface with Variable Baud-Rate: Architecture and Proof-of-Concept
}

\author{
Arnaud Dupas, Patricia Layec, Eric Dutisseuil, Sergio Belotti, Sébastien Bigo, \\ Emilio Hugues Salas, Georgios Zervas, Dimitra Simeonidou.
}

\begin{abstract}
Varying the symbol rate is an alternative or complementary approach to varying the modulation format or the channel spacing, in order to turn optical networks into elastic networks. We propose to allocate just-enough bandwidth for each optical connection by adjusting the symbol rate such that penalty originating from long cascades of optical filters is contained. This helps reducing overprovisioning for lightpaths where full capacity is not needed, by (i) eliminating unnecessary regenerators and (ii) reducing the power consumption of terminals, when the clock rate of electronics is reduced along with the Baud rate. We propose a novel architecture for an elastic optical interface combining a variable bitrate transceiver, paired with an elastic aggregation stage, with software-defined control. We then report a real-time FPGA-based prototype, delivering flexible transport frames to be sent with a PDM-QPSK modulation format. We interconnect this prototype with a commercial OTN switch and a centralized controller. We demonstrate fast and hitless reconfiguration of the interface and measure the reconfiguration time of hardware logic $(<450 \mu \mathrm{s})$ as well as end-to-end control and data plane $(<0.9 \mathrm{~s})$.
\end{abstract}

Index Terms-Elastic Optical Networks; Optical communication; Software Defined Networking; Flexgrid

\section{INTRODUCTION}

$\mathbf{T}$ The commercial availability of $100 \mathrm{G} / 200 \mathrm{G}$ and $400 \mathrm{G}$ transponder cards answers to the need for high transported capacity [1]. To achieve this, higher order modulation formats and faster symbol rates are two popular means which are often combined. Therefore intermediate regenerations are more and more required and are not only used for long distance links [2]. To further lower the cost per

Manuscript received June 17, 2016.

A. Dupas, P. Layec; E. Dutisseuil, S. Bigo are with Nokia Bell Labs France (corresponding author: arnaud.dupas@nokia.com).

S. Bellotti is with Nokia Italy.

E. Hugues Salas, G. Zervas, and D. Simeonidou are with the High Performance group, University of Bristol, UK. bit, optical networks have to be rethought and in particular have to adapt to traffic demands and transmission impairments.

Flexible networks are a promising way to improve the network resource utilization in wavelength division multiplexed optical networks. A first mean is the usage of Optical Transport Network (OTN) switching to improve the wavelength utilization, hence spectrum resources, up to an ideal matched of a fully utilized wavelength [3]. Elastic Optical Networks (EON) is another popular mean with several degrees of freedom [4][5][6]. In particular, the use of flexible frequency spacing between channels is advantageous for saving scarce optical spectrum and for improving network capacity [7]. This is usually referred to as a flexgrid scenario [8]. Variable symbol rate could also be used with fixed channel spacing such as $37.5 \mathrm{GHz}$ grid to contain filtering issue. With Nyquist WDM, signal is much less sensitive to crosstalk but still suffers from tight filtering and laser frequency detuning [9]. With OFDM, filtering impact and guard band were investigated in [10]. Recent works proposed to mitigate the filter penalty cascade with spectral engineering [11][12]. In this work, we rather consider that the real filling of channels is far from approaching $100 \%$. We thus proposed a just-enough bandwidth allocation by varying the symbol rate, hence the optical spectrum of each connection.

Typical application examples are inter-datacenter or metro networks which exhibit variable aggregated traffic for back-up services or between day and night operations. Along this path, in 2011 a real-time bandwidth-variable transponder (BVT) prototype was proposed in [13] and reduced the power consumption proportionally to the effective traffic load thanks to a flexible aggregation of Ethernet frames. Alternatively, a real-time board in [14] aggregating Ethernet traffic was demonstrated for an adaptive-modulation format transmission. To make reconfigurable EON a reality, hitless BVT becomes an important feature. In [15] a hitless code rate change was introduced to change the optical line net bit rate according to OSNR conditions, but no previous works have tackled the symbol rate variation which is of key interest for next generation of networks with more and more intermediate regenerations.

In this paper, we detail how a real-time hitless elastic optical interface is capable of aggregating the incoming 
client traffic based on tributaries (e.g. OTN or Ethernet) onto a just-enough bandwidth for each optical connection by adjusting the symbol rate [16]. The elastic optical interface creates flex-framing (e.g. flexible OTU signals [18]) and delivers the just-enough bitrate to take advantage of lower bandwidth occupancy as we keep the channel spacing constant. This helps reducing over-provisioning of lightpaths when full capacity is not needed and containing filtering cascade penalty. The proof-of-concept is based on a real-time FPGA prototype and commercial OTN switch which has been presented in [17]. These network elements are under the supervision of an open SDN controller, which is well-suited for an easy and precise management of elastic optical transmission according to light path requirements and impairments.

The rest of the paper is organized as follows: Section II details the flex framing architecture and review existing standards; Section III and IV detail the proof-of-concept together with obtained results characterizing the proposed hitless behavior and end-to-end reconfiguration to SDN controller. Finally Section V draws some conclusions of this work.

\section{ARChitecture of Elastic OpticAl InTERFACE}

The elastic optical interface not only delivers an adapted data rate in the WDM line interface, but also is capable of selecting the appropriate number of active clients' lanes upon the reception of SDN control message. This multilayer capability allows (i) to better fill the optical pipes and (ii) to adjust the optical pipe to the exact traffic demand with flexible aggregation and variable symbol rate.

The OTN standard contains in its digital layer several sub-layers: among them there is the optical channel digital unit (ODU) which encapsulates the client frame and the optical transport unit (OTU) corresponds to the resulting line rate since it adds forward error correction. The ODU sub-layer already exhibits some flexibility with either ODU multiplexing that allows to encapsulate low order ODU into higher order ODU or ODUFlex(GFP) for which the aggregated flow is mapped into a resizable container [18] to fill the gap of the ODU hierarchy. Now, flexibility is also needed at the OTU sub-layer to bridge the gap between transported line rate and flexible ODU hierarchy. The OTN standard is now currently under discussion to provide more flexibility at the OTU sub-layer for beyond 100G [19]. This will fully leverage on the flexible transmission parameters of the physical layer in order to optimize simultaneously the data rate, the reach and spectrum occupancy.

The beyond 100G OTN new frame is called OTUCn, embedding a variable parameter $\mathrm{n}$ representing the number of $100 \mathrm{G}$ units, and would be able to carry the expected $400 \mathrm{GbE}$ client when $n=4$. However, the line interfaces are not necessarily a multiple of $100 \mathrm{G}$, thus there is a possibility to describe this kind of frame as an OTUCn-M, where $n$ is still the number of $100 \mathrm{G}$ unit and $M$ the number of 5G tributary slots supported. As an example the 150G signal could be transported in an OTUC2-30 since it only carries 30 tributary slots out of the 40 that would be carried by a full 200G OTUC2. However, until the OTN standard is not completed, this might change. The Ethernet standard is also actively discussing the flexible framing with FlexE as analyzed in [20] and both the Ethernet and OTN standards evolutions are closely linked to help elastic optical networks become more dynamic with more automated functions. Target completion date for beyond 100G OTN standard or FlexE standard is about 2018/2020.

In the elastic optical interface prototype, we implemented the OTUCn concept, but scaled down to low speed due to real-time FPGA maximum speed. Indeed, the best evaluation board is capable of running at $~ 30 \mathrm{GBaud}$ with Xilinx Virtex 7 series, hence $\sim 100 \mathrm{Gbit} / \mathrm{s}$ for a PDMQPSK signal. We interleave a variable number $n$ of $10 \mathrm{~Gb} / \mathrm{s}$ frames (i.e. OTU2 frames). In Fig. 1, we illustrate the implemented concept in real-time for $n=5$. We can see that each OTU2 flow is split into the 4 output lanes and interleaved at the byte level with the others active OTU2 flows. We define a universal marker of the form "x.y" ( $\mathrm{x}$ and $y$ are integers where $x \in[0: 9] ; y \in[0: 3])$ and we insert these markers at regular intervals. This allows to decouple the number of $n$ varying input client flows from the number of physical output lanes (in PDM-QPSK, we have 4 output lanes corresponding to the two quadratures and polarizations). At the reception, the markers also permit the identification of the received data which help the skew alignment procedure.
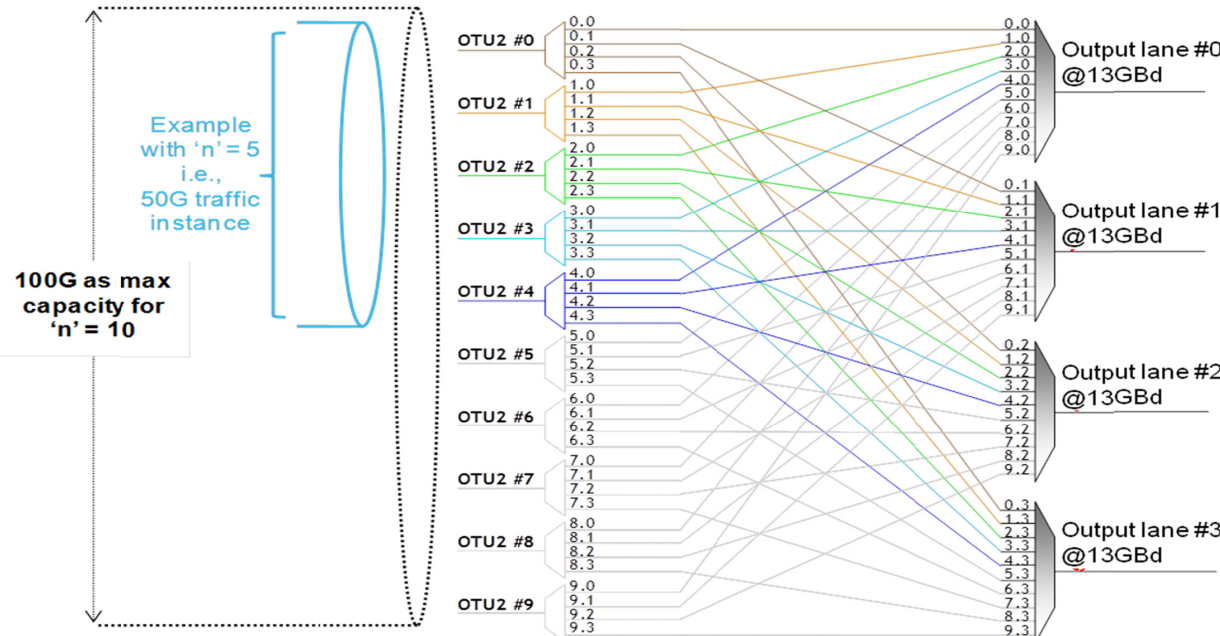

Fig. 1: Flexible aggregation principles when 5 input OTU2 flows are active out of 10 . The output lanes are then running at half the maximum baudrate. 


\section{PROOF-OF-CONCEPT}

To validate the variable symbol rate concept, we implemented a transmitter prototype based on programmable electronic (FPGA), electro-optical conversion, and reconfigurable with SDN controller and restful server. It has been characterized from end-to-end up to the off-line receiver combined with digital processing [16][17].

The elastic optical interface is associated with the aggregation function of an OTN switch, both controlled by only one SDN controller (Fig. 2). To realize this function, we developed a restful server, which is able to interpret the commands of a SDN controller like OpenDayLight [25] or ONOS [26] via a restful API.

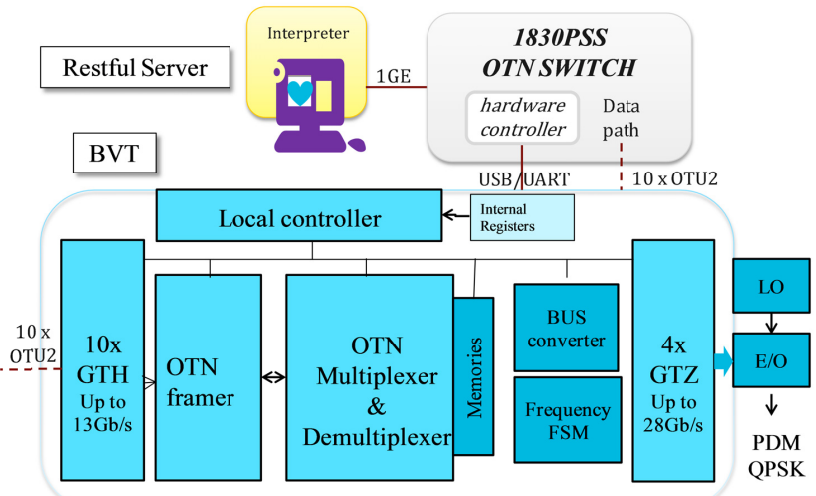

Fig. 2: Architecture of Elastic Optical Interface with controller

The incoming signaling messages are in http/json format and can be sent by a smartphone or a application dedicated to optical control. They include the information related to frequency slot via nominal central frequency with parameter $\mathrm{n}$ and slot width with parameter $\mathrm{m}$ to be multiplied to $12.5 \mathrm{GHz}$ as defined in [8] and [27] to be occupied by the elastic optical signal. The restful server has the knowledge of the hardware state composed of the OTN switch and the elastic optical interface. When a new frequency demand has been interpreted and is compatible with transmitter capabilities, low level commands are sent via $1 \mathrm{GE}$ link to the hardware controller of the OTN switch matrix 1830PSS-36. The linux kernel of this hardware controller has been modified to adapt the commands coming from restful server into registers access orders with handchecking. Inside the BVT box (Fig. 2), a register map is programmed and used by a local controller to perform all

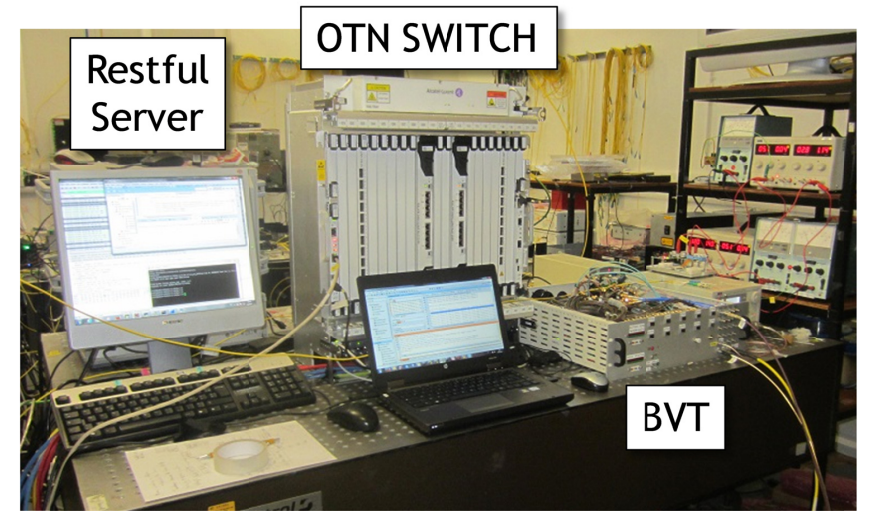

Fig. 3: Experimental set-up. the switching, memories and Finite State Machine control. It is able also to manage the number of OTU2 lanes at 10.7 Gbit/s that has to be multiplexed and demultiplexed and to control the baudrate of the high speed interfaces in order to generate the appropriate OTUFlex-like multiplexed flow. As the output baudrate can vary from $2.67 \mathrm{GBd}$ to $26.7 \mathrm{GBd}$ to generate a multiplexed optical signal from $10.7 \mathrm{Gbit} / \mathrm{s}$ to 107 Gbit/s via a PDM-QPSK electro-optical modulator, the Restful server sends the corresponding number of slots $\mathrm{m}$, with $m=1,2$ or 3 . Thus the maximum spectral occupancy in terms of flexi-grid slots is $37.5 \mathrm{GHz}$ for this experiment and this granularity of $12.5 \mathrm{GHz}$. This can be easily adapted to higher bitrates and finer granularities.

In addition to the flexible aggregation and symbol rate, we propose a hitless protocol storing the frames into on-chip memory when needed. This allows to demonstrate the first elastic optical transmitter which can adapt its output bitrate when input frames are added or dropped, without service disruption or packet loss. The hitless protocol relies on a Finite State Machine as described in Fig. 4, where the packets flow is stopped after OTN multiplexing. The local controller manages the finite state machine and informs the Restful server when the reconfiguration is finished. Thus the restful server can accept a new bandwidth request.

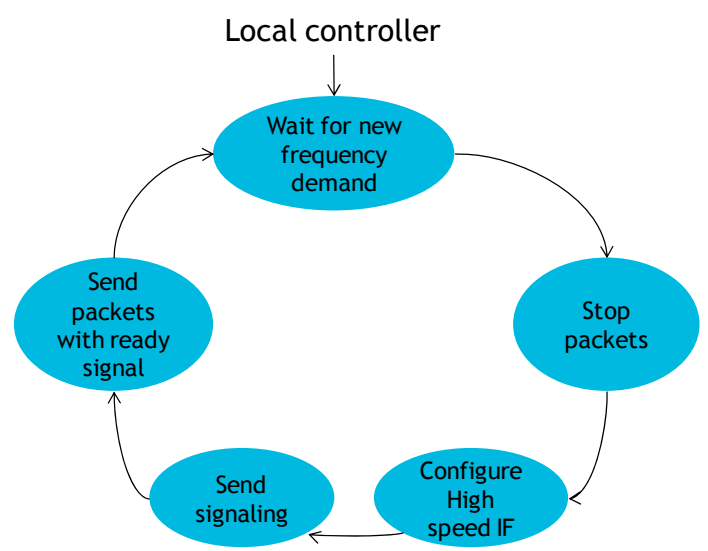

Fig. 4: Finite State Machine for hitless behaviour.

The control of the Elastic Optical Interface has been performed from an OpenDayLight SDN controller via the restful server in the context of multi-level and multi-domain network environment [28].

\section{EXPERIMENTAL RESULTS}

The optical output of the BVT has been sent to an optical spectrum analyzer and an off-line receiver composed of an optical coherent mixer with a local oscillator, a series of 4 high speed $40 \mathrm{GHz}$ photodiodes and a digital oscilloscope with a $3 \mathrm{~dB}$ bandwidth of $20 \mathrm{GHz}$ of and an acquisition rate of 40 GSps. The digital processing is performed off-line on the stored data to recover the PDM-QPSK constellation and measure the Bit Error Rate on the interleaved frames

The BVT has been first characterized optically with commands coming from its local controller. In Fig. 5, we see the optical spectrum of the PDM-QPSK signal for 2 to 10 OTU2 clients managed by the local controller, corresponding to an output bitrate of, respectively $21.4 \mathrm{Gbit} / \mathrm{s}$ to $107 \mathrm{Gbit} / \mathrm{s}$. 
We see directly the impact on the spectral occupancy.

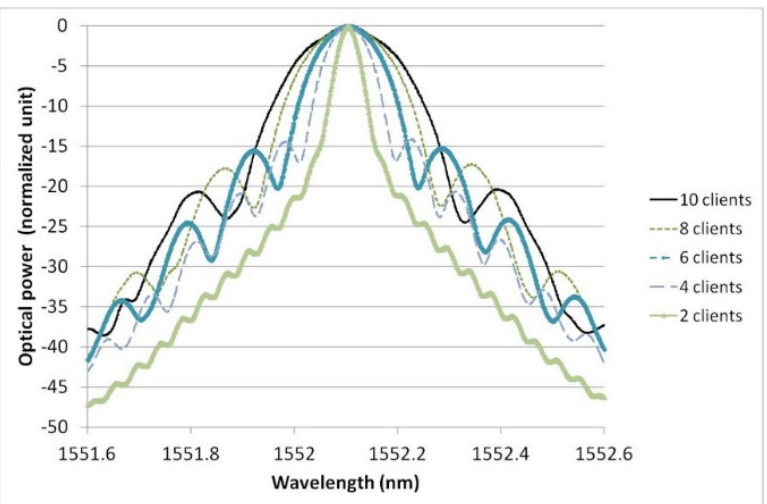

Fig. 5: Optical spectrum at BVT output after electro-optical modulation for different OTU2 client numbers and Baud rates.

Fig. 6 represents the electronic eye diagrams for two different configurations. With 10 OTU2 clients at the input of the transmitter, the total output bitrate is maximum with $107 \mathrm{Gbit} / \mathrm{s}$ corresponding to a one I/Q electrical signal at 26.7 GBauds (Fig. 6a). Fig. 6b shows the eye diagram when 4 OTU2 clients are multiplexed byte by byte, resulting in a 42.8 GBauds electrical signal on one I/Q arm of the modulator.
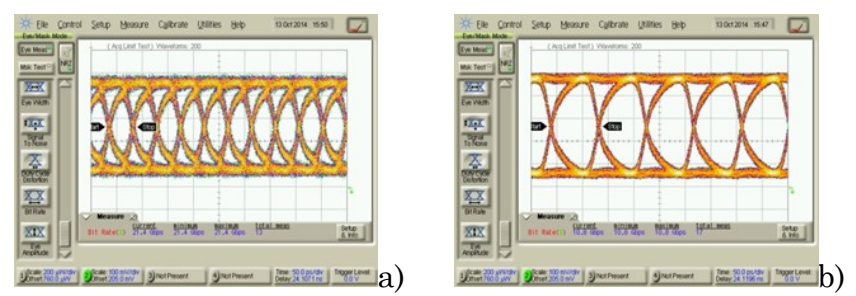

Fig. 6: BVT electrical outputs before E/O conversion for 10 clients (a) and 4 clients (b).

The BVT has been then characterized in terms of quality of transmission and switching time when a new frequency command arrives at the restful server. The switching time displayed in Fig. 7 is the measured time of the total reset sequence of high speed output serializer/deserializer interfaces (GTZ in Xilinx Virtex 7), in which the serializer is aligned, reconfigured and no data is transmitted. This switching time includes $\sim 2 \mu$ s guard time, in which the reference clock for the data is unstable [23]. It is technology dependent and may be improved with the most recent programmable electronic technologies and ASIC progress. Fig. 7 illustrates all combinations of any starting bitrate to ending bitrate, both from $10.7 \mathrm{Gbit} / \mathrm{s}$ to $107 \mathrm{Gbit} / \mathrm{s}$, corresponding to the number of multiplexed OTU2 clients, which is an integer in the range [1:10]. In all 100 configurations the switching time is below $450 \mu \mathrm{s}$ and mainly depends of the ending bitrate. For example, case a $(423 \mu \mathrm{s})$ in Fig. 7 is the measured time of the switching from $75 \mathrm{Gbit} / \mathrm{s}$ to $107 \mathrm{Gbit} / \mathrm{s}$. Case b ( $432 \mu \mathrm{s})$ is the switching time from $107 \mathrm{Gbit} / \mathrm{s}$ to $53.5 \mathrm{Gbit} / \mathrm{s}$. During the reconfiguration phase, the high speed transmitters are reset, and when it's finished, notification signal is sent to the local controller, which can start again the packets transmission. A maximum pause time of $450 \mu$ s on the data flow corresponds to a maximum of 37 OTU2 frames to be stored and delayed in on-chip memories, which represents $74 \%$ of the total available block memories in this FPGA whitout need of external memory.

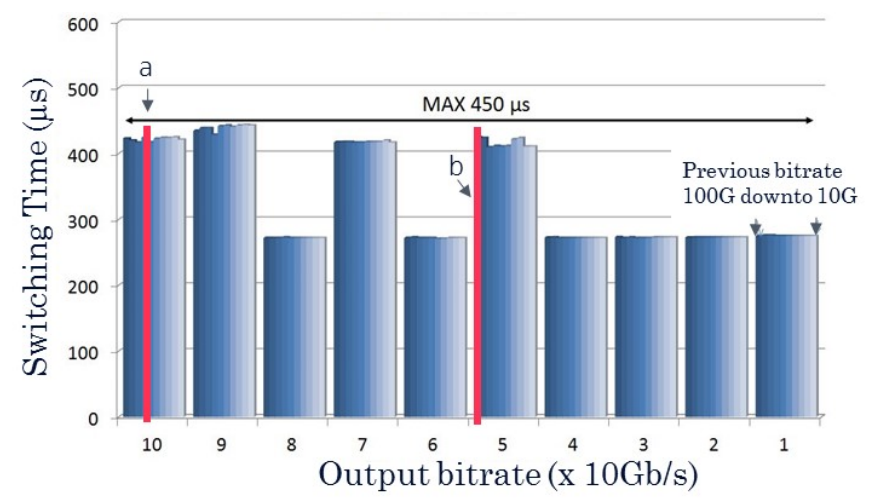

Fig. 7: Switching time for all starting and ending bitrates combinations (10x10 in total).

When the configuration for the new frequency request is done, the stored frames can be sent again, avoiding the lost of any data, but with additional latency due to the traffic interruption. This latency is temporary because it can be absorbed after reconfiguration by a real-time adjustment of the buffer emptying and a synchronization with the knowledge of the real traffic load. Latency variation is also limited to the reconfiguration time and the buffer reading, and can be then cancelled due to synchronous and deterministic processing inside the FPGA. With the management of the QoS of the traffic inside the OTN switch and an accurate monitoring of the OTU2 frames number to be multiplexed and transported, service continuity can be performed without any packet loss.

The quality of the elastic optical signal has been measured with the Bit Error Rate vs the OSNR in 0.1nm for different transmitted bitrates (Fig. 8). The OSNR penalty for a Q-Factor of $8.5 \mathrm{~dB}$ (HD-FEC limit) remains below $1.1 \mathrm{~dB}$ for the bitrates of $107 \mathrm{Gbit} / \mathrm{s}$ and $75 \mathrm{Gbit} / \mathrm{s}$, compared to the theoretical limit for a PDM-QPSK signal. This is partially due to the skew between the IQ lines of the two polarizations which are not easy to tune in this FPGA prototype. The constellation after offline processing for the two polarization states is inserted in Fig. 8 for the maximum transmitted bitrate of $107 \mathrm{Gbit} / \mathrm{s}$ and has been recorded in the laboratory clear and open for all combinations of bitrates. 


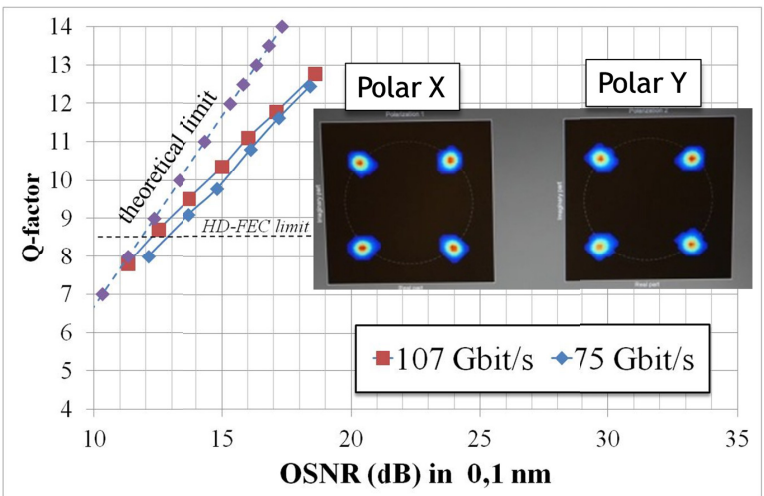

Fig. 8: BER measurement in back-to-back transmission at $107 \mathrm{~Gb} / \mathrm{s}$ and $75 \mathrm{~Gb} / \mathrm{s}$.

We now evaluate the total duration for a line-rate reconfiguration, including the request from the controller. In Fig. 9, we can observe, at the restful server level, with Wireshark the necessary control messages to select a new bitrate. From the moment when a new http request is made (zero time ${ }^{*} \mathrm{REF}^{*}$ reference), the answer to SDN controller is found to return after $0.89 \mathrm{~s}$ in the worst configuration of Fig. 7 with a http OK answer. During this period of time, the BVT was reconfigured in less than $450 \mu \mathrm{s}$ without interruption of service and the hardware controller has checked and sent back the state of the BVT and the OTN switch. With an adapted planning tool and a synchronization with the control of flexible and configurable optical cross connects as presented in [28], we can manage from end-to-end an optical path with variable bitrate and optimize the spectral efficiency in a multi-wavelength environment.

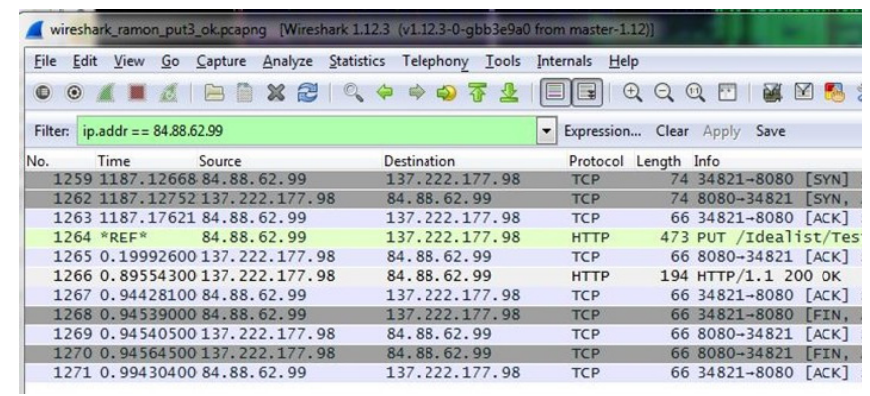

Fig. 9: Control messages at restful server level for bit rate change.

- $\quad$ curl -H 'Content-type: application/json' -X PUT http://137.222.177.98:8080/XXX/ -d '\{"modFormat"="DP-QPSK", "modFactor"="4", "symRate"="13.4", "lineRate"="53.5", "freqSlot"="2"\}'

Fig. 10: Control messages at restful server level for bit rate change.

As an example, Fig. 10 shows the messages received at the restful server level which IP address is 137.222.177.98. The JSON data model includes different information relevant to a transmitter among them the symbol rate, the line rate, and the frequency slot are applied straight forward to our BVT.

\section{CONCLUSiON}

An Elastic Optical Transponder with variable baudrate can also bring advantages in complement with format variable transponders in the frame of Elastic Optical Networks. We presented the first integration of an elastic Bandwidth Variable Transmitter (BVT) with a restful server and a commercially available OTN switch operating up to 107 Gbit/s. In all bitrate configurations, the switching time was measured below $450 \mu \mathrm{s}$ with an OSNR penalty below $1.1 \mathrm{~dB}$ at HD-FEC limit for two configurations. With a restful API, the BVT can be configured and monitored to be fully compliant with the last defined flexi-grid ITU-T standards and a fine bandwidth accuracy of $12.5 \mathrm{GHz}$. This can lead to the deployment of Bandwidth on Demand applications where no traffic interruption is required, and to a better spectral resources management in Elastic Optical Networks.

The adoption of elastic optical interface also depends on the expected benefits in a real network.

\section{ACKNOWLEDGMENT}

This work was partly supported by FP7 EU project IDEALIST under grant agreement $n^{\circ} 317999$. We thank our colleagues and partners for the fruitful discussions and meetings we had during these last three years.

\section{REFERENCES}

[1] B. Lavigne, O. Bertran-Pardo, C. Bresson, M. Lefrancois, E. Balmefrezol, M. Le Monnier, L. Raddatz, L. Suberini, "400Gb/s Real-time Trials on Commercial Systems for Next Generation Networks," in Journal of Lightwave Technology., Sept. 2015.

[2] O. Rival, A. Morea, "cost-efficiency of mixed 10-40-100 Gb/s networks and elastic optical networks", in Proc. OFC, paper OTuI4, 2011

[3] V. Hutcheon, "OTN to enable flexible networks," in Proc OFC, paper NMC1, 2011.

[4] M. Jinno, H. Takara, Y. Sone, K. Yonenaga, A. Hirano, "Multiflow optical transponder for efficient multilayer optical networking," in IEEE Commun. Mag., vol. 50, no.5, May 2012.

[5] O. Gerstel, M. Jinno, A. lord, S.B. Yoo, "Elastic optical networking: A new dawn for the optical layer?," in IEEE Commun. Mag., vol. 50, no.2, 2012

[6] P. Layec, A. Morea, F. Vacondio, O. Rival, and J.-C. Antona "Elastic Optical Networks: The Global Evolution to Software Configurable Optical Networks," in Bell Labs Technical Journal, vol. 18, no.3, pp. 133-151, Dec. 2013.

[7] A. Morea, J. Renaudier, T. Zami, A. Ghazisaeidi, O. BertranPardo, "Throughput comparison between $50 \mathrm{GHz}$ and $37.5 \mathrm{GHz}$ grid transparent networks," in J. of Opt. Commun. Netw., vol.7, no. 2, Feb. 2015.

[8] ITU-T recommendation "Spectral grids for WDM applications: DWDM frequency grid," G.694.1, Feb. 2012, www.itu.int

[9] A. Ghazisaeidi, P. Tran, P. Brindel, O. Bertran-Pardo, J. Renaudier, G. Charlet, and S. Bigo, "Impact of tight optical filtering on the performance of 28 Gbaud Nyquist-WDM PDM8QAM over 37.5 GHz grid," in Proc. OFC, paper OTu3B.6, 2013.

[10] B. Kozicki H. Takara, T. Yoshimatsu, K. Yonenaga, M. jinno, "Filtering Characteristics of Highly-Spectrum Efficient Spectrum-Sliced Elastic Optical Path (SLICE) Network," in Proc OFC, paper JWA43, 2009.

[11] T. Rahman, A. Napoli, D. Rafique, E. de Man, M. Bohn, C.M. Okonkwo, H. de Waardt, "Mitigation of filtering cascade 
penalties using spectral shaping in optical nodes" in Proc ECOC, paper P4.19, 2014.

[12] O. Bertran Pardo, T. Zami, B. Lavigne, M. Le Monnier, "Spectral engineering technique to mitigate $37.5-\mathrm{GHz}$ filter cascade penalty with real-time 32-GBaud PDM-16QAM," in Proc OFC, paper M3A3, 2015.

[13] F. Vacondio, A. El Falou, A. Voicila, C. le Bouëtté, J.-M. Tanguy, C. Simonneau, E. Dutisseuil, J.-L. Pamart, L. Schoch, O. Rival, "Real-time elastic coherent muxponder enabling energy proportional optical transport," in Proc OFC, paper JTh2A.51, 2013.

[14] S. Yan, Y. Yan, B.R. Rofoee, Y. Shu, E. Hugues-Salas, G. Zervas, D. Simeonidou, "Demonstration of Real-Time Ethernet to Reconfigurable Superchannel Data Transport over Elastic Optical Network," in Proc ECOC, paper Mo.4.2.3, 2014.

[15] F. Cugini, F. Paolucci, F. Fresi, G. Meloni, G. Berrettini, N. Sambo, A. Giorgetti, T. Foggi, L. Poti, P. Castoldi, "Benefits of active stateful PCE for flexgrid networks," in Proc. OFC, 2014.

[16] A. Dupas, P. Layec, E. Dutisseuil, S. Bigo, S. Belotti, S. Misto, S. Annoni ,Y. Yan, E. Hugues-Salas, G. Zervas, D. Simeonidou, "Hitless 100 Gbit/s OTN Bandwidth Variable Transmitter for Software-Defined Networks," in Proc. OFC, paper TH3I.1, 2016

[17] A. Dupas, E. Dutisseuil, P. Layec, P. Jennevé, S. Frigerio, Y. Yan, E. Hugues-Salas, G. Zervas, D. Simeonidou, S. Bigo, "Real-Time Demonstration of Software-Defined Elastic Interface for Flexgrid Networks," in Proc. OFC, paper M3A, 2015

[18] ITU-T Standardization Sector, "Interfaces for the Optical Transport Network," ITU-T Rec. G.709/Y.1331, Edition 4.2, Feb. 2012.

[19] ITU-T Study Group 15, http://www.itu.int/en/ITUT/studygroups/2013-2016/15

[20] S J. Trowbridge, "Ethernet and OTN - 400G and beyond", in Proc. OFC, Th3H.1, 2015

[21] A. Carena, V. Curri, G. Bosco, P. Poggiolini, F. Forghieri, "Modeling of the impact of nonlinear propagation effects in uncompensated optical coherent transmission links," in J. Light. Technol., May 012.

[22] A. Lord, et al., "Elastic Optical Network Architecture: Reference scenario, cost and planning," IDEALIST deliverable D1.1, June 2013.

[23] A. Dupas, et al., "Real-Time Demonstration of SoftwareDefined Elastic Interface for Flexgrid Networks," in Proc.OFC 2015, paper M3A.2.

[24] F. Cugini, et al., "Benefits of active stateful PCE for flexgrid networks," in Proc. of OFC, 2014.

[25] OpenDayLight foundation, www.opendaylight.org

[26] ONOS consortium, http://onosproject.org

[27] IETF draft (passed LC for RFC informational) draft-ietfccamp-flexi-grid-fwk "Framework and Requirements for GMPLS-based control of Flexi-grid DWDM networks", O.Gonzales de Dios,R. Casellas, 2015.

[28] O. Gonzales de Dios et al., "First Demonstration of Multivendor and Multi-domain EON with S-BVT and Control Interoperability over Pan-European testbed", in Proc. ECOC 2015, Post-deadline paper PDP4.1, Valencia, 2015. 